\title{
Integrable Operators and Canonical Differential Systems
}

\author{
Lev Sakhnovich \\ My address: Lev Sakhnovich, 735, Crawford, Brooklyn, 11223,New York, \\ NY, USA. \\ My tel. (718) 646-2757. \\ My e-mail address:Lev.Sakhnovich@verizon.net \\ Research Professor,University of Connecticut at Storrs.
}




\begin{abstract}
In this article we consider a class of integrable operators and investigate its connections with the following theories:the spectral theory of non-self-adjoint operators, the Riemann-Hilbert problem, the canonical differential systems and the random matrices theory.
\end{abstract}

\title{
Introduction
}

In the article [12] we considered the operators of the type

$$
S f=L(x) f(x)+P . V . \int_{a}^{b} \frac{D(x, t)}{x-t} f(t) d t,
$$

where $f(x) \in L_{k}^{2}(a, b)$ and $k \times k$ matrix functions $L(x)$ and $D(x, t)$ are such that

$$
L(x)=L^{\star}(x), \quad D(x, t)=-D^{\star}(t, x) .
$$

(The symbol P.V. indicates that the corresponding integral is understood as the principal value.)

Later in the work [8] the important class of the operators $\mathrm{S}$, when

$$
k=1, \quad L(x)=1, \quad D(x, x)=0
$$

was studied in details. These results had a number of interesting applications $[5],[8]$.

In our works [12],[13] the connection of the operators $\mathrm{S}$ with the spectral theory of non-selfadjoint operators was shown. The operator identity

$$
(Q S-S Q) f=\int_{a}^{b} D(x, t) f(t) d t, \quad Q f=x f(x)
$$

plays an essential role in these articles. From the identity (4) follows the statement. 
Proposition 1. Let the kernel $D(x, t)$ be degenerate, i.e. $D(x, t)=i A(x) A^{\star}(t)$, where $A(x)$ is a $k \times m$ matrix function $(k \leqslant m)$. If the operator $S$ is invertible, then the operator $T=S^{-1}$ has the form

$$
T f=M(x) f(x)+P . V . \int_{a}^{b} \frac{E(x, t)}{x-t} f(t) d t,
$$

where $M(x)=M^{\star}(x)$ and the kernel $E(x, t)$ is also degenerate and has the form

$$
E(x, t)=i B(x) B^{\star}(t)
$$

$B(x)$ is a $k \times m$ matrix function.

The operators $S$ and $T$ lead to the Riemann-Hilbert matrix problem

$$
W_{+}(\sigma)=W_{-}(\sigma) R^{2}(\sigma), \quad a \leqslant \sigma \leqslant b
$$

where $m \times m$ matrix function $W(z)$ is analytic, when $z \notin[a, b]$. Here matrix function $R^{2}(\sigma)$ can be constructed with the help of the operators $S$ and $T, W_{ \pm}(\sigma)$ is defined by the relation

$$
W_{ \pm}(\sigma)=\lim W(z), \quad y \rightarrow 0, \quad z=\sigma+i y
$$

In the present article an essential role is played by the canonical differential system

$$
\frac{d}{d x} W(x, z)=i \frac{J H(x)}{z-x} W(x, z), \quad W(0, z)=I_{m},
$$

where $m \times m$ matrix $J$ is such that

$J=J^{\star}, \quad J^{2}=I_{m}$ and $H(x) \geqslant 0$.

The monodromy matrix of system (9) coincides with the solution of the RiemannHilbert problem (7), i.e.

$$
W(z)=W(b, z)
$$


Let us note that $W(z)$ is a characteristic matrix function of the operator (see $[2],[10])$

$$
A f=x f+i \int_{a}^{x} \beta(x) J \beta^{\star}(t) f(t) d t, \quad f(x) \in L_{k}^{2}(a, b),
$$

where $\beta(x)$ is a $k \times m$ matrix function such, that

$$
\beta^{\star}(x) \beta(x)=H(x) .
$$

We deduce in this article a new sufficient condition of the linear similarity of the operator $A$ to the operator $Q f=x f$. It easily follows from (9) that $W(x, z)$ in the neighborhood of $z=\infty$ admits the representation

$$
W(x, z)=I_{m}+\frac{M_{1}(x)}{z}+\frac{M_{2}(x)}{z^{2}}+\ldots,
$$

where

$$
M_{1}(x)=i \int_{a}^{x} J H(t) d t .
$$

In view of (9) and (14) all the coefficients $M_{k}(x)$ are defined if the coefficient $M_{1}(x)$ is known. This fact is of interest as the representation

$$
W(b, z)=I_{m}+\frac{M_{1}(b)}{z}+\frac{M_{2}(b)}{z^{2}}+\ldots
$$

is closely connected with the problems of the random matrices theory [4],[14]. From the view point of the random matrix theory it is important that in this article the procedure of constructing the matrix $M_{1}(x)$ is given (section 3 ). We pay the principal attention to the matrix version of the class (3), when

$$
k \geqslant 1, \quad L(x)=I_{k}, \quad D(x, x)=0 .
$$

For this class the corresponding matrix function $R^{2}(x)$ from $(7)$ has a special structure, namely

$$
\left[R^{2}(x)-I_{m}\right]^{2}=0
$$


The corresponding matrix function $J H(x)$ is nilpotent when $m=1$, i.e.

$$
[J H(x)]^{2}=0
$$

In the last part of the paper we consider a number of examples.

\section{Integrable operators and Riemann-Hilbert prob- lem}

In this section we remind of a number of facts contained in the paper [12]. We use these facts in the next sections. Let $W(z)$ be $m \times m$ matrix function.

We suppose that the following conditions are fulfilled.

1). Matrix function $W(z)$ is analytic in the domain $z \notin[a, b],(-\infty<a<b<\infty)$ and satisfies the equality

$$
W(z)=I_{m}+\frac{1}{2 \pi i} \int_{a}^{b} \frac{F(x)}{x-z} d x
$$

where $F(x)$ is bounded $m \times m$ matrix function on the segment [a,b].

2). The relations

$$
\begin{gathered}
W^{\star}(z) J W(\bar{z})=J, \\
i \frac{W^{\star}(z) J W(z)-J}{z-\bar{z}} \geqslant 0, \quad z \neq \bar{z}
\end{gathered}
$$

are true. ( Here $m \times m$ matrix $J$ satisfies the equalities $J=J^{\star}, \quad J^{2}=I$ ). The equality (1) guarantees the almost everywhere existence of the limits

$$
W_{ \pm}(x)=\lim W(z) \quad \text { as } \quad y \rightarrow \pm 0, \quad z=x+i y
$$

Now we use the polar decomposition (see [11])

$$
W_{+}(x)=U(x) R(x), \quad W_{-}(x)=U(x) R^{-1}(x),
$$


where $m \times m$ matrix functions $U(x)$ and $R(x)$ are such that

$$
U^{\star}(x) J U(x)=J, \quad J R(x)=R^{\star}(x) J
$$

and in addition the spectrum of $R(x)$ is positive.

Matrix function $R(x)$ is called $J$-module of matrix function $W_{+}(x)$. By relations (23) and (24) we have

$$
R^{2}(x)=J W_{+}^{\star}(x) J W_{+}(x) .
$$

According to the theory of J-module [11] the relations

$$
\begin{gathered}
D(x)=J\left[R(x)-R^{-1}(x)\right] \geqslant 0, \quad x \in[a, b], \\
D(x)=0, \quad x \notin[a, b]
\end{gathered}
$$

are true. Now we introduce the matrix functions $F_{1}(x), F_{2}(x)$ with the help of the relations

$$
F_{1}^{\star}(x) F_{1}(x)=D(x), \quad F_{2}(x)=F_{1}(x) J U^{\star}(x) .
$$

Remark 1. Matrix functions $F_{1}(x)$ and $F_{2}(x)$ are $k \times m$ matrices, where $k=$ $\sup [\operatorname{rank} D(x)], a \leqslant x \leqslant b$. Hence $k \leqslant m$.

Using relations (23),(26) and (28) we can write

$$
W_{+}(x)-W_{-}(x)=F_{2}^{\star}(x) F_{1}(x)=F(x) .
$$

In addition to conditions 1) and 2) we suppose:

$3)$. The matrix functions $F_{1}(x)$ and $F_{2}(x)$ are bounded on segment [a,b].

Let us define the operators $\Pi$ and $\Gamma$ by formulas $\Pi g=\frac{1}{\sqrt{2 \pi}} F_{1}(x) g$, $\Gamma g=-\frac{i}{\sqrt{2 \pi}} F_{2}(x) g$, where $g$ are $m \times 1$ vectors, $\Pi g$ and $\Gamma g$ belong to $L_{k}^{2}(a, b)$. Then we have

$$
\Pi^{\star} f(x)=\frac{1}{\sqrt{2 \pi}} \int_{a}^{b} F_{1}^{\star}(x) f(x) d x
$$




$$
\Gamma^{\star} f(x)=\frac{i}{\sqrt{2 \pi}} \int_{a}^{b} F_{2}^{\star}(x) f(x) d x,
$$

where $f(x) \in L_{k}^{2}(a, b)$. The next assertion follows from formulas (19),(30) and (31).

Proposition 2. The matrix function $W(z)$ admits the realization

$$
W(z)=I_{m}-\Gamma^{\star}(Q-z I)^{-1} \Pi
$$

where the operator $Q$ is defined by the relation

$$
Q f=x f, \quad f(x) \in L_{k}^{2}(a, b) .
$$

Next we introduce the $k \times k$ matrix

$$
L(x)=\left[I_{k}+\frac{1}{4}\left(F_{1}(x) J F_{1}^{\star}(x)\right)^{2}\right]^{1 / 2}
$$

and consider the operators

$$
\begin{aligned}
& S f=L(x) f(x)+\frac{i}{2 \pi} P . V \cdot \int_{a}^{b} \frac{F_{1}(x) J F_{1}^{\star}(t)}{x-t} f(t) d t, \\
& T f=L(x) f(x)-\frac{i}{2 \pi} P . V . \int_{a}^{b} \frac{F_{2}(x) J F_{2}^{\star}(t)}{x-t} f(t) d t .
\end{aligned}
$$

The introduced operators $\mathrm{S}$ and $\mathrm{T}$ are acting in the space $L_{k}^{2}(a, b)$ and $f(x)$ is a $k \times 1$ vector function.

Theorem 1.(see [13], p.45-46) The operators $S$ and $T$ are positive, bounded and

$$
T=S^{-1}, \quad S F_{2}(x)=F_{1}(x) J
$$

From relation (23) we deduce that

$$
W_{+}(x)=W_{-}(x) R^{2}(x), \quad x \in[a, b]
$$




$$
W_{+}(x)=W_{-}(x), \quad x \notin[a, b]
$$

Formulas (38) and (39) lead to the Riemann-Hilbert Problem.

Problem 1. To recover the matrix function $W(z)$ by the given J-module $R(x)$. In the case $J=I$ Problem 1 plays an essential role in the prediction theory of the stationary processes [15]. The case when $J \neq I$ is important for the theory of random matrices [5], [8],[14].

We solve Problem 1 in the following way.

1.By the given matrix $R^{2}(x)$ we construct the matrix $D(x)$ (see $(26)$ ).

2.Using the first of equalities $(28)$ we find $F_{1}(x)$.

3. With the help of formula (1) the operator $S$ is constructed.

4.Due to the second equality of (37) we have $F_{2}(x)=S^{-1} F_{1}(x) J$.

5.Now it is easy to see that formulas (19) and (29) give the solution of the Riemann-Hilbert problem (7) with the normalizing condition

$$
W(z) \rightarrow I \quad \text { as } \quad z \rightarrow \infty
$$

Remark 2. The operators $S$ and $T$ defined by formulas (1) and (5) are called integrable [5], [8]. The case when $k=1$ and

$$
F_{1}(x) J F_{1}^{\star}(x)=0
$$

has important applications in the theory of the random matrices(see [4], [7], [8], [14]). The general case was used in the spectral theory of the non-selfadjoint operators [12],[13]. 


\section{Spectral theory}

We introduce some important notions .

Let the linear bounded operator have the form

$$
A=A_{R}+i A_{I}
$$

where $A_{R}$ and $A_{I}$ are self-adjoint operators acting in Hilbert space $H$.There is a bounded linear operator $K$ which maps a Hilbert space $G$ in $H$ so that

$$
A_{I}=K J K^{\star}
$$

where $J$ acts in $G$ and $J=J^{\star}, \quad J^{2}=I$.

Definition 2 (see [2], [10)]. The operator function

$$
W(\lambda)=I-2 i K^{\star}(A-\lambda I)^{-1} K J
$$

is called the characteristic operator function of $A$.

We recall that the simple part of $A$ means the operator which is induced by $A$ on the subspace $H_{1}=\overline{\sum_{k=0}^{\infty} A^{k} D_{A}}$, where $D_{A}=\overline{\left(A-A^{\star}\right) H}$. In paper [12] we deduced Theorem 1 for the case $m \leqslant \infty$. From this fact we obtain the following assertion [12],[13].

Theorem 2. If the characteristic operator function $W(z)$ of the operator $A$ satisfies the condition

$$
\|W(z)\| \leqslant c, \quad z \neq \bar{z}
$$

for some $c$, then the simple part of $A$ is linearly similar to a self-adjoint operator with a absolutely continuous spectrum

It follows from relation (45) that $W(z)$ satisfies the conditions 1)-3). The converse is not true. Using this fact we receive a new version of Theorem 2. 
Theorem 3. If the characteristic operator function $W(z)$ of the operator $A$ satisfies the conditions 1)-3), then the statement of Theorem 2 is true.

Example. We consider the case when

$$
F_{1}(x)=[x+i, x-i], \quad 0 \leqslant x \leqslant 1, \quad j=\left[\begin{array}{cc}
-1 & 0 \\
0 & 1
\end{array}\right]
$$

. The corresponding operator $S$ has the form

$$
S f=f(x)-\frac{1}{\pi} \int_{0}^{1} f(t) d t .
$$

Due to relations (46) and (47) we have

$$
F_{2}(x)=[-q(x), \overline{q(x)}],
$$

where

$$
q(x)=x+\frac{1}{2(\pi-1)}+i \frac{\pi}{\pi-1} .
$$

Using the property of the Cauchy integral (see[6]) we deduce from relation (19) that

$$
\begin{gathered}
W(z)=-\frac{1}{2 \pi i} F(0) \log z+0(1), \quad z \neq \bar{z}, \quad|z|<\frac{1}{2}, \\
W(z)=-\frac{1}{2 \pi i} F(1) \log (z-1)+0(1), \quad z \neq \bar{z}, \quad|z-1|<\frac{1}{2} .
\end{gathered}
$$

It follows from formulas (46) and (48),(49) that $F(0) \neq 0, \quad F(1) \neq 0$. Hence the constructed $W(z)$ satisfies the conditions of Theorem 3 but does not satisfy the condition (45) of Theorem 2.

\section{Canonical differential systems}

It follows from Theorem 3 that the following operator

$$
S_{\xi} f=L(x) f(x)+\frac{i}{2 \pi} P . V . \int_{a}^{\xi} \frac{F_{1}(x) J F_{1}^{*}(t)}{x-t} f(t) d t
$$


is positive, bounded and invertible.

We set

$$
\begin{gathered}
\Phi(\xi, x)=S_{\xi}^{-1} F_{1}(x), \\
B(\xi)=\frac{1}{2 \pi} \int_{a}^{\xi} \Phi^{*}(\xi, x) F_{1}(x) d x .
\end{gathered}
$$

Lemma 1. The matrix function $B(\xi)$ is absolutely continuous and monotonically increasing .

Proof. As it is known [3],[9] the operator $S^{-1}$ can be represented in the form

$$
S^{-1}=U^{\star} U,
$$

where the linear bounded operator $U$ acts in the space $L_{k}^{2}(a, b)$ and satisfies the condition

$$
U^{\star} P_{\xi}=P_{\xi} U^{\star} P_{\xi}, \quad a \leqslant \xi \leqslant b,
$$

where $P_{\xi} f(x)=f(x), a \leqslant x \leqslant \xi$ and $P_{\xi} f(x)=0, \quad \xi \leqslant x$. From relations (54) and (55) we deduce the equality

$$
\frac{d}{d x} B(x)=H(x)=\frac{1}{2 \pi} h^{\star}(x) h(x),
$$

where

$$
h(x)=U F_{1}(x) .
$$

The lemma is proved.

Let us consider the system of equations

$$
W(x, z)=I+i J \int_{a}^{x} \frac{d B(\xi)}{z-\xi} W(\xi, z) .
$$

Theorem 4. (see[13], Ch.3) The following equality

$$
W(b, z)=W(z)
$$


holds.

Corollary 1. The integral system (59) is equivalent to the differential system

$$
\frac{d W(x, z)}{d x}=\frac{i J H(x)}{z-x} W(x, z), \quad H(x) \geqslant 0
$$

with the boundary condition $W(a, z)=I_{m}$. Here the matrix function $H(x)$ is defined by relation (57).

Corollary 2. The matrix function $W(z)$ is the monodromy matrix of system (61), i.e. $W(z)=W(b, z)$.

Due to (61) in the neighborhood of $z=\infty$ the following relation

$$
W(x, z)=I+M_{1}(x) / z+M_{2}(x) / z^{2}+\ldots
$$

is fulfilled. It follows from (59) and (61) that

$$
M_{1}(x)=i J B(x)
$$

Formulas (53) (54) and (63) give the solution of the following inverse problem. Problem 2. To recover the matrix function $H(x)$ and $M_{1}(x)$ by the given $J$ module $R(x)$. Theorem 3 and relation (54) imply the following assertion.

Proposition 3. If equality

$$
F_{1}(x)=0, \quad \alpha \leqslant x \leqslant \beta, \quad \alpha \neq \beta
$$

is true then

$$
F_{2}(x)=0, \quad W_{+}(x)=W_{-}(x), \quad R(x)=I, \quad \alpha \leqslant x \leqslant \beta .
$$

Corollary 3. If condition (64) is fulfilled then

$$
B^{\prime}(x)=H(x)=0, \quad \alpha \leqslant x \leqslant \beta .
$$




\section{Examples}

Example 1. Let us consider the case when

$$
J=j=\left[\begin{array}{cc}
-I_{m} & 0 \\
0 & I_{m}
\end{array}\right]
$$

and

$$
R^{2}(x)=\left[\begin{array}{cc}
0 & \phi(x) \\
-\phi^{\star}(x) & 2 I_{m}
\end{array}\right], \quad 0 \leqslant x \leqslant r,
$$

where $\phi(x) \phi^{\star}(x)=I_{m}$. From (68) we deduce that

$$
R(x)=1 / 2\left[\begin{array}{cc}
I_{m} & \phi(x) \\
-\phi^{\star}(x) & 3 I_{m}
\end{array}\right]
$$

The matrix $R(x)$ satisfies the following conditions.

1. The spectrum of $R(x)$ is positive.

Indeed, we obtain by direct calculation that $[R(x)-I]^{2}=0$. Hence the spectrum of the matrix $R(x)$ is concentrated at the point $\lambda=1$.

2. The relation

$$
j R(x)=R^{\star}(x) j
$$

is true.

It means that $R(x)$ is the $\mathrm{j}$-module of the matrix $W(z)$ which satisfies relation (7). From (68) we deduce that

$$
R(x)-R^{-1}(x)=\left[\begin{array}{cc}
-I_{m} & \phi(x) \\
-\phi^{\star}(x) & I_{m}
\end{array}\right]
$$

According to (71) we have

$$
D(x)=j\left[R(x)-R^{-1}(x)\right]=\left[\begin{array}{cc}
I_{m} & -\phi(x) \\
-\phi^{\star}(x) & I_{m}
\end{array}\right] .
$$


Hence the equality

$$
F_{1}(x)=\left[I_{m},-\phi(x)\right]
$$

holds. Using (73) we obtain the relations

$$
\begin{gathered}
F_{1}(x) j F_{1}^{\star}(x)=0, \\
F_{1}(x) j F_{1}^{\star}(t)=\phi(x) \phi^{\star}(t)-I_{m}
\end{gathered}
$$

Thus in case (69) we deduce from (52) and (74),(75) that operator the $S_{\xi}$ has the form

$$
S_{\xi} f=f(x)+\frac{i}{2 \pi} P . V . \int_{0}^{\xi} \frac{\phi(x) \phi^{\star}(t)-I_{m}}{x-t} f(t) d t .
$$

The fact that the operator $V$ defined as

$$
V f=\frac{1}{\pi} P . V . \int_{-\infty}^{\infty} \frac{f(t)}{x-t} d t, \quad f \in L^{2}(-\infty, \infty)
$$

is unitary implies that

$$
S_{\xi} \geqslant 0 \text {. }
$$

Further we suppose that the operator $S_{r}$ is invertible.

Hence the operators $S_{\xi}, \quad \xi \leqslant r$ are invertible as well.

Remark 3. If $\phi(x)$ satisfies Hölder condition then there exists such $r>0$ that $S_{r}$ is invertible.

Using relation (53) we have

$$
\Phi(x, \xi)+\frac{i}{2 \pi} P . V . \int_{0}^{\xi} \frac{\phi(x) \phi^{\star}(t)-I_{m}}{x-t} \Phi(t, \xi) d t=F_{1}(x) .
$$

where

$$
\Phi(x, \xi)=\left[\Phi_{1}(x, \xi), \Phi_{2}(x, \xi)\right]
$$


Here $\Phi_{k}(x, \xi)$ are $m \times m$ matrix functions $(k=1,2)$. It follows directly from (73) and (79) that

$$
\begin{gathered}
\Phi_{1}(x, \xi)+\frac{i}{2 \pi} P . V \cdot \int_{0}^{\xi} \frac{\phi(x) \phi^{\star}(t)-I_{m}}{x-t} \Phi_{1}(t, \xi) d t=I_{m}, \\
\Phi_{2}(x, \xi)+\frac{i}{2 \pi} P . V . \int_{0}^{\xi} \frac{\phi(x) \phi^{\star}(t)-I_{m}}{x-t} \Phi_{2}(t, \xi) d t=-\phi(x),
\end{gathered}
$$

and

$$
\Phi_{1}(x, \xi) \Phi_{1}^{\star}(x, \xi)=\Phi_{2}(x, \xi) \Phi_{2}^{\star}(x, \xi) .
$$

Due to (37) and (54) the formulas

$$
\begin{gathered}
F_{2}(x)=\left[-\Phi_{1}(x, 1), \Phi_{2}(x, 1)\right] \\
B(\xi)=\frac{1}{2 \pi} \int_{0}^{\xi}\left[\begin{array}{cc}
\Phi_{1}(x, \xi) & \Phi_{2}(x, \xi) \\
-\phi^{\star}(x) \Phi_{1}(x, \xi) & -\phi^{\star}(x) \Phi_{2}(x, \xi)
\end{array}\right] d x .
\end{gathered}
$$

are true.

Example 2. We separately consider the partial case of Example 1, when $m=1$.

It follows from (72) and (82) that

$$
\Phi_{2}(x, \xi)=-\phi(x) \overline{\Phi_{1}(x, \xi)} .
$$

Hence formula (85) takes the form:

$$
B(\xi)=\frac{1}{2 \pi} \int_{0}^{\xi}\left[\begin{array}{cc}
\Phi_{1}(x, \xi) & -\overline{\Phi_{1}(x, \xi)} \phi(x) \\
-\overline{\phi(x)} \Phi_{1}(x, \xi) & \overline{\Phi_{1}(x, \xi)}
\end{array}\right] d x .
$$

Comparing formulas (57) and (87) we deduce the representation

$$
H(x)=B^{\prime}(x)=a(x)\left[\begin{array}{cc}
1 & e^{i \alpha(x)} \\
e^{-i \alpha(x)} & 1
\end{array}\right],
$$


where $a(x) \geqslant 0, \quad \alpha(x)=\overline{\alpha(x)}$. Due to (88) the matrix $j H(x)$ is nilpotent, i.e.

$$
[j H(x)]^{2}=0
$$

Example 3. Let us consider the partial case of Example 1, when

$$
m=1, \quad \phi(x)=e^{2 i u x}, \quad u=\bar{u} .
$$

Example 3 plays an important role in the theory of the random matrices [4], [7],[14]. Now the operator $S_{\xi}$ takes the form

$$
S_{\xi} f=f(x)-\frac{1}{\pi} \int_{0}^{\xi} e^{i u(x-t)} \frac{\operatorname{sinu}(x-t)}{x-t} f(t) d t .
$$

The operator $S_{\xi}$ defined by formula (52) is invertible for all $0<\xi<\infty$ (see [4], p.167).

We denote by $\Psi(x, \xi, u)$ the solution of the equation

$$
\Psi(x, \xi, u)-\frac{1}{\pi} \int_{0}^{\xi} \frac{\sin u(x-t)}{x-t} \Psi(t, \xi, u) d t=e^{-i u x} .
$$

Then according to relations (81) and (82) we have

$$
\Phi_{1}(x, \xi, u)=e^{i u x} \Psi(x, \xi, u), \quad \Phi_{2}(x, \xi, u)=-e^{-i u x} \overline{\Psi(x, \xi, u)} .
$$

It follows from (87) and (93), that

$$
B(\xi, u)=\frac{1}{2 \pi} \int_{0}^{\xi}\left[\begin{array}{cc}
e^{i u x} \Psi(x, \xi, u) & -e^{i u x} \overline{\Psi(x, \xi, u)} \\
-e^{-i u x} \Psi(x, \xi, u) & e^{-i u x} \overline{\Psi(x, \xi, u)}
\end{array}\right] d x
$$

Example 4. Let us consider the case when $m=1$ and

$$
J=j=\left[\begin{array}{cc}
-1 & 0 \\
0 & 1
\end{array}\right]
$$


and

$$
R(x)=\frac{1}{2}\left[\begin{array}{cc}
2-|\psi(x)|^{2} & -\overline{\psi(x)}^{2} \\
\psi(x)^{2} & 2+|\psi(x)|^{2}
\end{array}\right], \quad 0 \leqslant x \leqslant r .
$$

The matrix $R(x)$ satisfies the following conditions.

1. The spectrum of $R(x)$ is positive.

Indeed, we obtain by direct calculation that $[R(x)-I]^{2}=0$. Hence the spectrum of the matrix $R(x)$ is concentrated at point $\lambda=1$.

\section{The relation}

$$
j R(x)=R^{\star}(x) j
$$

is true.

It means that $R(x)$ is the $\mathrm{j}$-module of the matrix $W(z)$ which satisfies relation (7). From (96) we deduce that

$$
R(x)-R^{-1}(x)=j D(x)=j F_{1}^{\star}(x) F_{1}(x),
$$

where

$$
F_{1}(x)=[\psi(x), \overline{\psi(x)}]
$$

Using (99) we obtain the relations

$$
\begin{gathered}
F_{1}(x) j F_{1}^{\star}(x)=0, \\
F_{1}(x) j F_{1}^{\star}(t)=\psi^{\star}(x) \psi(t)-\psi(x) \psi^{\star}(t)
\end{gathered}
$$

Thus we deduce from (99) and (101), that the operator $S_{\xi}$ in case (96) has the form

$$
S_{\xi} f=f(x)+\frac{i}{2 \pi} P . V . \int_{0}^{\xi} \frac{\psi^{\star}(x) \psi(t)-\psi(x) \psi^{\star}(t)}{x-t} f(t) d t .
$$

Further we suppose that the operators $S_{\xi}$ are positive and invertible $(0<\xi \leqslant r)$. Remark 4. If $\psi(x)$ satisfies the Hölder condition, then there exists such $r>0$ 
that the operators $S_{\xi}$ are positive and invertible $(0<\xi \leqslant r)$.

It follows directly from (99) and (102), that

$$
\begin{aligned}
& \Phi_{1}(x, \xi)+\frac{i}{2 \pi} P . V . \int_{0}^{\xi} \frac{\psi^{\star}(x) \psi(t)-\psi(x) \psi^{\star}(t)}{x-t} \Phi_{1}(t, \xi) d t=\psi(x), \\
& \Phi_{2}(x, \xi)+\frac{i}{2 \pi} P . V . \int_{0}^{\xi} \frac{\psi^{\star}(x) \psi(t)-\psi(x) \psi^{\star}(t)}{x-t} \Phi_{2}(t, \xi) d t=\overline{\psi(x)},
\end{aligned}
$$

where

$$
\Phi_{1}(x, \xi) \Phi_{1}^{\star}(x, \xi)=\Phi_{2}(x, \xi) \Phi_{2}^{\star}(x, \xi) .
$$

Due to (103) and (104) we have

$$
\begin{aligned}
& F_{2}(x)=\left[-\Phi_{1}(x, 1), \Phi_{2}(x, 1)\right], \quad \Phi_{1}(x, \xi)=\overline{\Phi_{2}(x, \xi)} \\
& B(\xi)=\frac{1}{2 \pi} \int_{0}^{\xi}\left[\begin{array}{ll}
\overline{\psi(x)} \Phi_{1}(x, \xi) & \overline{\psi(x) \Phi_{1}(x, \xi)} \\
\psi(x) \Phi_{1}(x, \xi) & \psi(x) \overline{\Phi_{1}(x, \xi)}
\end{array}\right] d x
\end{aligned}
$$

It follows from (107) that relation (89) is true in this case as well.

Remark 5. Comparing formulas (69) and (96) we see that Examples 1 and 3 coincide when $m=1$ and

$$
\phi(x)=-\overline{\psi(x)}^{2}, \quad|\phi(x)|=1 .
$$

Remark 6. If

$$
\psi(x)=i \sqrt{\gamma} e^{-i u x}, \quad 0<\gamma \leqslant 1
$$

due to (96) we have

$$
R^{2}(x)=\left[\begin{array}{cc}
1-\gamma & \gamma e^{2 i u x} \\
-\gamma e^{-2 i u x} & 2+\gamma
\end{array}\right]
$$

The corresponding Riemann-Hilbert problem was considered in [4].

Let us represent $\psi(x)$ in the form

$$
\psi(x)=A(x)+i B(x)
$$


where

$$
A(x)=\overline{A(x)}, \quad B(x)=\overline{B(x)} .
$$

Then the operator $S_{\xi}$ takes the form

$$
S_{\xi} f=f(x)-\frac{1}{\pi} P . V \cdot \int_{0}^{\xi} \frac{A(x) B(t)-B(x) A(t)}{x-t} f(t) d t .
$$

The following partial cases of $\psi(x)$ play an essential role in a number of applications [7]:

$$
\psi_{1}(x)=\sqrt{\pi}[A i(x)+i A i \prime(x)]
$$

where $A i(x)$ is the Airy function, and

$$
\psi_{2}(x)=\sqrt{\frac{\pi}{2}}\left[J_{\alpha}(\sqrt{x})+i \sqrt{x} J_{\alpha}^{\prime}(\sqrt{x})\right]
$$

where $J_{\alpha}(z)$ is the Bessel function.

Reference

1. Brodskii M.S., Triangular and Jordan Representation of Linear Operatorts, Amer.Math. Soc. 1971.

2. Brodskii M.S. and Livsic M.S., Spectral Analysis of Non-self-adjoint Operators and Intermediate Systems, Amer. Math. Soc. Transl.(2) 13, 265-346, 1960

3. Davidson K.R., Nest Algebras, Pitnam, Res. Notes Math.,1988

4. Deift P., Its A. and Zhou X, A Riemann-Hilbert Approach to Asymptotic Problems Arising in the Theory of Random Matrix Models, and also in the Theory of Integrable Statistical Mechanics, Annals of Math., 146, 149-235, (1997). 5. Deift P., Integrable Operators, Amer. Math. Soc. Transl. 2, v. 189, 69-84, 1999. 
6. Gakhov F.D., Boundary Problems, Nauka, Moscow, $197 \%$.

7. Harnad J.,Tracy C.A. and Widom H., Hamiltonian Structure of Equations Appearing in Random Matrices, arXiv, 1-18, 1993.

8. Its A.R.,Izergin V.E.,Korepin V.E. and Slavnov N.A., The quantum Correlation Function as the $\tau$ Function of Classical Differential Equations, 407417, Important developments in soliton theory, A.S.Fokas and V.E.Zakharov (eds)Springer Verlag, 1993.

9. Larson D. R., Nest Algebras and Similarity Transformation, Ann.Math., 125, p.409-427, 1985.

10. Livsic M.S.,Operators, Oscillations, Waves, Open Systems, Transl. of Math. Monographs, 34, Providence, 1973.

11. Potapov V.P.,The Multiplicative Structure of J-contractive Matrix Function, American Math. Society Translation, 15, 131-243, 1960.

12. Sakhnovich L.A., Operators Similar to the Unitary Operator with Absolutely Continuous Spectrum, Functional Anal. and Appl., 2:1, 48-60, 1968.

13. Sakhnovich L.A., Spectral Theory of Canonical Differential Systems.Method of Operator Identities. Operator Theory, Adv. and Appl.107, Birkhäuser, 1999. 14. Widom H., Asymptotic for the Fredholm Determinant of the Sine Kernel on a Union of Intervals, Comm. Math. Phys. 171, 159-180, 1995.

15. Wiener N.,Extrapolation, Interpolation and Smoothing of Stationary Time Series, Cambridge, 1949. 